\title{
MOUTH: A MIRROR OF BODY \& MIND
}

\author{
Dr. Rajalakshmi. G \\ MDS, PGDHM
}

\begin{abstract}
According to Williams Osler, Mouth is the mirror of the body, as mouth reflects many systemic diseases. Though the oral cavity can reflect and unravel many of the human body's internal secrets, in developing country like India most people don't tend to place the same importance on dental health as they do on their medical health. Systemic diseases can manifest oral symptoms at an early phase, which can be crucial for a diagnosis and timing of treatment. Areas such as the soft palate, hard palate, tongue, gingiva, oral mucosa, the dentition, periodontium, or the salivary gland tissue are usually affected. This article focuses on the importance of oral health and an overview on the oral manifestations of certain systemic and psychiatric disorders.
\end{abstract}

Key Words: Oral cavity, Oral manifestations, Systemic diseases, psychiatric disorders, Stress

\section{INTRODUCTION}

The mouth is the reflection of health or disease, a sentinel or early warning system. It is considered to be the entryway to the body and is the hub of a invariable brook of invaders like bacteria, viruses, parasites, and fungi, challenges the mouth. Many systemic diseases and psychiatric disorders have oral manifestations. These lesions usually develops on the oral mucosa, tongue, gingiva, dentition, periodontium, salivary glands, facial skeleton, extra oral skin and other related structures. Oral sign may be the first and sometimes even the only evidence of a disturbed state e.g. Koplik's spots in the buccal mucosa which precede the cutaneous eruption of measles. Many systemic and few stress related disorders show oral manifestations, so it must be properly documented for the patient to receive appropriate diagnosis and management. Though overlooked, oral cavity is an important diagnostic area not just because it contains derivatives of all of the primary germinal layers, but includes tissues not demonstrable anywhere else in the body. It also has role in diagnosing a number of systemic and psychiatric diseases. This article is a concise overview of the Stress related and Systemic oral manifestations as a wider appraisal is beyond the scope of this manuscript.

\section{STRESS RELATED ORAL MANIFESTATIONS}

The mouth is a mirror of health or disease, a sentinel or early warning system. As the gateway to the body, a constant barrage of invaders like bacteria, viruses, parasites, and fungi, challenges the mouth. Many systemic diseases have oral manifestations. These lesions develop on the oral mucosa, tongue, gingiva, dentition, periodontium, salivary glands, facial skeleton, extraoral skin and other related structures. These oral manifestations must be properly recognized if the patient is to receive appropriate diagnosis and referral for treat. Thus oral cavity is an important diagnostic area not just because it contains derivatives of all of the primary germinal layers, and includes tissues not demonstrable anywhere else in the body, but also because of its role played in diagnosing a number of systemic diseases just because of

their oral manifestations.

Stress is defined as a physical, mental or emotional response to events that causes bodily or mental tension ${ }^{1}$. The etiopathogenesis of many diseases are discovered and managed at any early stage, but some diseases are considered as idiopathic, hence diagnosis and treatment plan of such diseases always becomes controversial. Stress is one such etiology or predisposing factor in many diseases. Stress in related to hypertension, gastric ulcer and diabetes mellitus. Many schools of thought established and identified the role of stress as one of the etiological factor in oral lesions A few are described below ${ }^{1}$.

Lichen planus is a relatively common, chronic dermatologic disease that often affects the oral mucosa. Lichen planus is an immunologically mediated mucocutaneous disorder. Skin lesions are classically described as purple, pruritic, polygonal papules usually affecting the flexor surface of extremities ${ }^{2}$. Oral Lichen Planus(OLP) may present as reticular, erosive or bullous type. Reticular lichen planus is common and it involves the buccal mucosa, lateral and dorsal surface of tongue, gingiva, palate and vermilion border ${ }^{3}$. Typical radiating white striae and erythematous atrophic mucosa are present at the periphery of well-demarcated ulcerations on the posterior buccal mucosa. Topical steroids applied to most symptomatic areas are usually significant ${ }^{4}$.

Atrophic or erosive OLP involving the gingiva is often referred to as desquamative gingivitis, a descriptive clinical term used for bright red edematous patches involving the full width of the attached gingiva. Symptomatic treatment can be provided by topical analgesics or antihistamine rinses or more specifically by use of topical steroids ${ }^{4}$.

Apthous ulcers are recurrent oral ulcerations. It is the most common disease affecting the oral mucosa which is often characterized by the appearance of one or more painful ulcers which heal after a few days or weeks, but may recur after a 


\section{International Journal of Engineering Applied Sciences and Technology, 2019 \\ Vol. 4, Issue 7, ISSN No. 2455-2143, Pages 165-168 \\ Published Online November 2019 in IJEAST (http://www.ijeast.com)}

variable period of time. There are three types namely minor apthous ulcers, major apthous ulcers and herpetiform ulcers ${ }^{2}$. The etiology of these ulcers has not been clearly established but emotional stress and cessation of smoking are implicated. There is considerable evidence that immune responses are involved in the pathogenesis of these recurrent ulcers. Tetracycline mouthwash and topical steroid application can be used for treating apthous ulcers ${ }^{3}$.

Burning mouth syndrome is associated with burning sensation of tongue, lips and other mucosal surfaces. Predominatly seen in females. Post - menopausal symptoms are significantly high. Sleep disturbances are common. Treatment needs to be tailored according to the etiological factors identified in an individual patient. Symptomatic relief and management of any associated behavioral or psychiatric disorders may be considered.

Myofacial pain is referred from a localized tender area, a trigger point in a taut band of skeletal muscles of the body including the muscles of mastication. Stress has a significant cause for clenching and grinding habits, resulting in spasm of the muscles of mastication ${ }^{1,6}$. Occlusal abnormalities also play a role in the etiology of the pain syndrome. MPDS is primarily a result of emotional rather than occlusal and mechanical factors.

Glossopyrosis: Burning tongue and burning sensations of the oral mucosa are common. ${ }^{3}$

Chronic periodontal diseases (CPD): Emotional factors also probably play a significant role in the complex etiology of $\mathrm{CPD}$, in which the gingival and underlying periodontal tissue cannot respond adequately to the local irritation of bacterial plaque and calculus.

Necrotizing gingivitis: ANUG is a fusospirochetal infection caused by local and systemic predisposing factors. Among these, emotional stress appears to be the most common etiology. ${ }^{3,6}$

Xerostomia:. Xerostomia is the abnormal reduction of saliva. ${ }^{3}$ Saliva plays a significant role in the maintenance of oral and general health.

Erythema multiforme (EM) and mucous membrane pemphigoid (MMP) In EM and MMP, emotional stress plays a major role in determining the severity of the disease as well as recurrent episodes. Remissions and exacerbations of long standing MMP are often related to stress and emotional instability ${ }^{3}$.

Bruxism: Grinding teeth is a neurotic habit that usually occurs during sleep. It may result in wear of teeth, trauma to periodontal tissues, and occasionally facial musculature and temporomandibular joint structures. ${ }^{3}$

Anorexia \& bulimia nervosa: The dentists and dental hygienists may be the first health care providers to assess the physical and oral effects of anorexia nervosa and bulimia nervosa. Bulimia nervosa is an eating disorder. It causes the affected individual to binge eat and then exercise excessively or induce vomiting to make up for the extra calories. This disorder is usually a result of an underlying psychotic issue Biting of oral mucosa (self mutilation) Biting of oral mucosa or tongue can induce severe trauma.

Atypical facial pain: Patients suffering from these pains are frequently described as having underlying psychiatric disturbances. $^{3}$

\section{SYSTEMIC ORAL MANIFESTATIONS}

Systemic oral manifestations may be briefed under nutritional, hematologic, gastrointestinal, renal, cardiovascular, endocrine, metabolic and dermatologic disorders.

\section{NUTRITIONAL DEFICIENCIES}

Vitamin A: Eruption rate is retarded, alveolar bone formation affected, hyperplastic gingival epithelium followed by keratinization.

Vitamin D: Developmental anomalies of dentin and enamel, delayed eruption, and misalignment of the teeth in the jaws. Vitamin D resistant rickets often associated with periapical involvement of normal appearing deciduous or permanent teeth, followed by the development of multiple gingival fistulas. Radiographically there is abnormal alveolar bone pattern. Cementum, and lamina dura around the teeth is absent or poorly defined.

Hypophosphatasia: premature loss of deciduous teeth, primarily the incisors.

Vitamin K: Most common oral manifestation is gingival bleeding. Prothrombin levels below 35\%, results in bleeding after tooth brushing; and below $20 \%$ result in spontaneous gingival hemorrhages.

Vitamin C deficiency (Scurvy): Inflammation of the interdental and marginal gingival followed by bleeding, ulceration, foul breaths. Hemorrhages and swelling of the periodontal membranes occur, tracked by loss of bone and loosening of the teeth, which eventually exfoliate.

Riboflavin deficiency: Glossitis involving the tip and/or the lateral margins of the tongue, leading atrophy of papillae. Tongue may turn magenta color. Pallor, involving oral mucosa followed by cheilosis, maceration and fissuring at the angles of the mouth.

Niacin deficiency: Oral mucosa becomes fiery red and painful. Glossitis, pain, redness and ulceration begin at the interdental papillae and spread rapidly.

\section{HEMATOLOGICAL DISORDERS}

Anaemia: Iron deficiency anemia is the most common hematologic disorder with characteristic oral manifestations including atrophic glossitis, mucosal pallor, and angular cheilitis. 
Plummer Vinson syndrome is associated which includes dysphagia due to pharyngoesophageal ulcerations.

Sickle cell anemia associated mandibular salmonella osteomyelitis that results in areas of osteoporosis and erosion followed by osteosclerosis. Anesthesia or paresthesia of the mandibular nerve may be seen.

Thalaseemia: Reports of oral complications from the thalassemias are rare in the literature. Pain and swelling in the parotid glands of patients with thalassemia major have been reported.

Leukemia: Oral complications of leukemia frequently include gingival hypertrophy, petechiae, ecchymosis, mucosal ulcers, and hemorrhage. Rarely associated with mental nerve neuropathy, called "numb chin syndrome"

Multiple myeloma: It is a plasma cell dyscrasis in which there is overproduction of specific immunoglobulins. Multiple myeloma involving the oral cavity is usually a late secondary manifestation of lesions in the jaws, commonly mandible. These lesions cause swelling of the jaws, pain, numbness, mobility of teeth, and pathological fractures. Punched out lesions of the skull and jaw are the hallmark radiographic findings.

\section{METABOLIC AND ENDOCRINE DISORDERS}

Diabetes mellitus: Associated with xerostomia, resulting in increase susceptibility to opportunistic infections like Candida albicans Concomitant diffuse, non tender, bilateral enlargement of the parotid glands, called diabetic sialadenoses. Other manifestation includes Erythematous candidiasis, mucormycosis, benign migratory glossitis, altered taste and burning mouth syndrome.

Hyperparathyroidism: Radiographically loss of the lamina dura surrounding the roots of the teeth is an early manifestation of hyperparathyroidism, with alterations in the jaw trabecular pattern. Also shows a decrease in trabecular density, and blurring of the normal pattern resulting in a "ground glass" appearance radiographically. "Brown tumor" of hyperparathyroidism may develop progressivly.

Hypoparathyroidism:, If occurs during odontogenesis or tooth development, a pitting enamel hypoplasia and failure of tooth eruption may occur. The presence of persistent oral candidiasis in a young patient may be a indicator for the onset of endocrinecandidiasis syndrome.

Hypoadrenocorticism: or addison's disease. Orofacial manifestations include "bronzing" or hyper pigmentation of skin, especially in sun-exposed areas and over pressure points. Skin changes are often preceded by oral mucosal melanosis. The diffuse or patchy brown macular pigmentation commonly occurs on the buccal mucosa, floor of the mouth, ventral tongue, and other areas of the oral mucosa.

Hypercortisolism: The patient may present with a variable degree of facial hirsutism. Pathological fractures of the mandible, maxilla or alveolar bone may occur upon low impact trauma due to osteoporosis. Healing of fractures and extraction socket delayed

\section{DERMATOLOGIC DISEASES}

Lichen planus: The oral manifestations of lichen planus may occur weeks or months before the appearance of the skin lesions. The oral lesions are characterized by presence of striae of Wickham ${ }^{3}$.

Pemphigus: A vesiculobullous lesion in which bullae tend to rupture as soon as they form. The oral mucosa also elicit Nikolsky's phenomenon.

Cicatricial pemphigoid: Characterized by vesiculobullous lesions, which are relatively thick walled and, may persist for 24 to 48 hours before rupturing and desquamating.

Psoriasis: Characterized by angular cheilosis, fissured tongue and benign migratory glossitis. Lesions involve the lips, buccal mucosa, palate, gingiva and floor of the mouth. Appear as gray or yellowish-white plaques, silvery white scaly lesions with an erythematous base. Also as multiple papular eruptions, which may be ulcerated; or as small, papillary, elevated lesions with a scaly surface.

Erythema multiforme: Oral manifestations include hyperemic macules, papules or vesicles, which may become eroded or ulcerated and bleed freely. The tongue, palate, buccal mucosa and gingiva are commonly involved.

Stevens Johnsons syndrome: Oral lesions may be extremely severe. Mucosal vesicles or bullae occur which rupture and leaves surfaces covered with a thick white or yellow exudate. The lips may exhibit ulcerations with bloody crusting and are painful.

Epidermolysis bullosa dystrophica: Characterized by oral bullae which on rupturing results in pain and scar formation. Dental defects consist of rudimentary teeth, congenitally absent teeth, hypoplastic teeth and crowns denuded of enamel ${ }^{7}$.

\section{GASTROINTESTINAL DISEASES}

Crohn's disease: Oral lesions have been documented to precede the intestinal lesions by years.In some cases is the only manifestation of the disease.Clinically, may present with diffuse swelling of one or both lips associated with angular cheilitis, hyperplastic rigid mucosa and "cobble stoning" of the buccal mucosa $a^{7,13}$.

Gastroesophageal Reflux (GER) is a common condition in childhood characterized by the rise of gastric contents into the esophagus. For extra-esophageal syndromes, dental erosions and Sandifer syndrome are considered conditions related to GERD $^{12}$. Acid reflux at the level of the oral cavity, in fact, can cause the dissolution of the tooth enamel, especially at the level of the palatal surfaces of the posterior teeth ${ }^{7}$.

Ulcerative colitis has been associated with destructive oral ulcerations resulting from immune-mediated vasculitis.. Pyostomatitis vegetans characterized by multiple painless 


\section{International Journal of Engineering Applied Sciences and Technology, 2019 \\ Vol. 4, Issue 7, ISSN No. 2455-2143, Pages 165-168 \\ Published Online November 2019 in IJEAST (http://www.ijeast.com)}

intraepithelial micro abscesses connecting in linear or serpentine tracks mostly on the labial mucosa, soft palate and ventral tongue may be seen ${ }^{7}$.

Celiac disease: This is a chronic intestinal disease caused by intolerance to gluten associated with poor digestion and absorption of the majority of nutrients and vitamins, which may affect both developing dentition and oral mucosa. Dental erosion involves lingual erosion on the palatal surfaces of the maxillary teeth with a smooth, glossy appearance and increased tooth sensitivity ${ }^{14}$

Renal Disease Uremic stomatitis has become relatively rare Painful plaques and crusts are distributed predominantly on the buccal mucosa, dorsum of the tongue, and floor of the mouth.

\section{CONNECTIVE TISSUE DISEASES}

Sjogren's syndrome: characterized by dry mouth (xerostomia), keratoconjunctivitis sicca, and other collagen diseases-often rheumatoid arthritis ${ }^{8}$.

Scleroderma: Scleroderma is a chronic disease characterized by diffuse sclerosis of the skin, gastrointestinal tract, heart muscles, lungs, and kidney. The lips of a patient with scleroderma may appear to be pursed due to constriction of the mouth aperture, thus making it difficult to open the mouth. The oral mucosa appears pale and feels rigid. The tongue can lose mobility and become smooth with flattening of the palatal rugae. Salivary hypo function can also be present ${ }^{8}$.

Lupus erythematosus: The oral lesions in the discoid form begin as erythematous areas, usually depressed, and typically with white spots or radiating white striae. Occasionally, superficial, painful ulceration may occur with crusting or bleeding. The central healing may result in depressed scarring ${ }^{8}$.

Rheumatoid arthritis: Temporomandibular joint (TJM) is often involved in rheumatoid arthritis. This is usually characterized by erosions in the condyle leading to a decreased activity of the mandible with pain upon movement. Oral dryness and salivary gland swelling can also be found in patients with rheumatoid arthritis ${ }^{8,13}$.

Cardiovascular Disorders Medications used for management of hypertension have various oral manifestations. Diuretics and adrenergic inhibitors may cause oral dryness and ulcerations. Calcium antagonists give rise to gingival overgrowth while ACE inhibitors may cause loss of taste and lichenoid reactions of the oral mucosa ${ }^{7}$.

Moreover various viral, bacterial and fungal infections show oral manifestations and mouth is the doorway of these organisms to our body ${ }^{15}$.

\section{CONCLUSION}

The oral manifestations empower the dentist to predict the systematic status of patients, thus identification of underlying undiagnosed disorder. Dentists must acquire acquaintance with systemic and psychiatric conditions that can affect the oral cavity, so that appropriate referral can be made. Moreover this article throws a light to the public regarding the importance of oral health. Thus Mouth is the Mirror of Human Body, so Oral Health Care a Must.

\section{REFERENCE}

1. T.N.Uma maheswari and N.Gnanasundaram Stress related oral diseases- a research study International Journal of Pharma and Bio Sciences Vol.1/Issue-3/Jul-Sep.2010

2. Neville B W, Damm D D, Allen C M, Bouquot J E. Relation of stress and anxiety to Oral Lichen planus .Oral Surg Oral Med Oral Pathol, 61:44, (1986).

3. Mc Cartan B. Pscychological factors associated with OLP J Oral Pathol Med, 24:273, (1995). Epstein JB. The Mouth: Window on systemic disease. Can Fam Physician1980 26: 953-7.

4.Brightman V J. Red and White lesions of oral mucosa In: Burket's Oral Medicine Diagnosis and Treatment, 9th Edn, J.P.Lippincott company, Philadelphia, (1994), pp.99-107

5. Brightman V J. Oral symptoms without apparent physical abnormality In: Burket's Oral Medicine Diagnosis and Treatment, 9th Ed. J.P.Lippincott Company, Philadelphia, (1994), pp.369-39

6. Travell J. Temporom andibular joint pain referred from muscles of head and neck. J Prosthet Dent, 10:745, (1960

7. Long RG, Hlousek L, Doyle JL. Oral manifestations of systemic diseases. Mt Sinai J Med 1998; 65: 309-15

8.. Shafer's Textbook of Oral Pathology. 4thed. Oral aspects of metabolic disease. In: WB.Saunders, 1993: p. 616-72.

10.Blood Dyscrasias Caused by Hypersensitivity to Drugs. Aster R.H. Pichler WJ (ed): Drug Hypersensitivity.

11Basel, Karger, 2 Williams AJ, Wray D, Ferguson A. The clinical entity of orofacial Crohn's disease. Q J Med 1991; 79: 451-8. 18.

12Beitman RG, Frost SS, Roth JLA. Oral manifestations of gastrointestinal disease. Dig Dis Sci 1981; 26: 741-7. 19.

13.Tyldesley WR. Mouth lesions as markers of gastrointestinal disease. Practitioner 1983; 227: 587-90. 007: p. 306-20.

14.. Cheraskin E. Oral manifestations of systemic diseases. J Natl Med Assoc 1958; 50: 241-7

15.Shafers W G, Hine M K, Levy B M, Tomich C E. Bacterial, viral, mycotic infection In : A Text book of Oral Pathology, 4th Edn, W B Saunders, Philadelphia, (1993), pp.368373. 12 